\title{
Lymphedema Development and Lymphatic Function following Lymph Node Excision in Sheep
}

\author{
Dalia Tobbia $^{\mathrm{b}}$ John Semple ${ }^{\mathrm{b}}$ Amy Baker $^{\mathrm{a}}$ Dan Dumont ${ }^{\mathrm{c}}$ Adam Semple $^{\mathrm{a}}$ \\ Miles Johnston ${ }^{a}$ \\ ${ }^{a}$ Neuroscience Program, Department of Laboratory Medicine and Pathobiology, Sunnybrook Health Sciences \\ Centre, ${ }^{\mathrm{b}}$ Department of Surgery, Women's College Hospital, and ${ }^{\mathrm{c} M o l e c u l a r}$ and Cell Biology, Department of \\ Medical Biophysics, Sunnybrook Health Sciences Centre, University of Toronto, Toronto, Ont., Canada
}

\section{Key Words}

Lymphedema $\cdot$ Lymph flow $\cdot$ Lymphangiogenesis •

Microlymphatics

\begin{abstract}
Background: Our objective was to develop an animal model of postsurgical lymphedema that would permit quantitation of edema and lymphatic function after the removal of a single popliteal lymph node in sheep. Methods: As a measure of lymph transport, ${ }^{125}$-human serum albumin was injected into prenodal vessels at 8,12 and 16 weeks after nodal excision, and plasma levels of the protein tracer were used to calculate the transport rate of the tracer to blood (percent injected per hour). Edema was quantified from the circumferential measurement of the hind limbs. Results and Conclusions: Following nodal excision, the limbs became progressively more edematous up to 3 days after nodectomy. After this, the swelling decreased but had not resolved even at 16 weeks after surgery. Compared with control limbs (17.2 $\pm 0.6 ; n=7)$, lymphatic function was depressed at 8 weeks after surgery $(10.6 \pm 1.5 ; n=7)$. At 12 $(14.4 \pm 1.0 ; n=7)$ and 16 weeks (13.9 $\pm 1.0 ; n=6)$, regeneration of lymphatic vessels at the excision site helped to restore about $80 \%$ of lymphatic capacity. These techniques
\end{abstract}

may be helpful in understanding the pathophysiology associated with cancer-related postsurgical lymphedema and may facilitate the development of new strategies to treat or prevent this condition.

Copyright $\odot 2009$ S. Karger AG, Basel

\section{Introduction}

The lymphedema associated with breast cancer-related lymph node dissection poses many challenges for health care professionals. If left untreated, the edema can lead to recurring infections, impaired limb function, psychosocial problems and, in extreme cases, malignant complications and life-threatening infections [1]. Treatment options for these patients have been very limited. Most individuals are offered some form of nonsurgical external compression therapies. This is achieved by the application of compression garments, the use of intermittent or sequential pneumatic compression devices and massage therapy. These approaches have met with some success but remain controversial and are far from ideal especially with entrenched, chronic edema [2].

Since the actual cause of the lymphedema is still being debated, the most appropriate target for new therapeutic 
approaches is not entirely apparent. It is generally accepted that damage to the lymphatics is involved in the pathogenesis. This has led to the assumption that stimulating new vessel growth is an appropriate therapeutic measure. Several groups are trying to achieve this by applying selected agents directly to affected tissues [3] or by introducing molecules through gene therapy approaches $[4,5]$. However, lymphatic vessels are routinely damaged in various other surgical procedures and lymphedema does not develop. What is generally not appreciated is the fact that lymphangiogenesis is a very vigorous process. Indeed, it has been difficult to develop chronic models of lymphostasis due to the remarkable regenerative capacity of the lymphatics and the development of collateral pathways around the site of obstruction [6]. In contrast, the removal of a lymph node or nodes appears to be a prerequisite for lymphedema development, although other factors such as radiotherapy in the affected limb may also be important [7].

While various mouse models have proven valuable in elucidating the molecular factors that control lymphatic vessel growth, due to their small size these animals are less suitable for analysis and quantitation of the physiological parameters that govern lymph flow after injury. In this regard, we know very little about the physiological properties of the newly formed vessels and how the transport capabilities of regenerating ducts are integrated functionally into a lymphatic network that includes lymph nodes as well as pre- and postnodal collectors. There would seem to be a need for the development of animal models of lymphedema that permit the quantitation of lymphatic function over time and allow assessment of the practical effectiveness of molecular and other forms of therapy.

With this in mind, lymphatic vessels in sheep may provide unique opportunities since the collecting ducts are relatively large and can be manipulated individually. In anticipation of studies that will investigate novel approaches to treat or prevent lymphedema, the objectives of this study were (1) to develop a model of lymphedema based on the removal of a single lymph node in sheep and (2) to quantify lymphatic transport over time and correlate this with edema formation.

\section{Materials and Methods}

A total of 41 randomly bred male and female Dorset sheep $(21.1-44.2 \mathrm{~kg})$ were used in this investigation. Animals were given free access to food and water for an observation period of 1 week preceding surgery. All experiments outlined in this paper were approved by the ethics committee at Sunnybrook Health Sciences Centre and conformed to the guidelines set by the Canadian Council on Animal Care and the Animals for Research Act of Ontario.

\section{Surgery}

Sheep were fasted $24 \mathrm{~h}$ prior to anesthesia. The animals were anesthetized initially with a 20 -ml i.v. injection of sodium pentothal. Subsequently, 2.0-3.5\% isofluorane was delivered through an endotracheal tube via a Moduflex (Dispomed) machine with a Hallowell respirator for surgical maintenance. The surgical site was shaved and washed with alcohol and betadine and then draped with sterile sheets. Sterile Evans blue dye (1\% in saline) was injected subcutaneously just proximal to the hoof to enhance visualization of the lymph node and the pre- and postnodal lymphatic vessels.

A vertical skin incision (approximately $8-10 \mathrm{~cm}$ long) was made over the lateral aspect of the popliteal region. The popliteal fossa is triangular in shape and is found by retracting the biceps femoris muscle caudally at the level of the stifle joint. At its distal angle, a single popliteal lymph node lies embedded in a pad of fat. The pre- and postnodal lymphatic vessels were tied off with a silk suture and the node was excised. Hemostasis was ensured prior to closure of the surgical site. The animals were returned to their holding pens after recovery from the anesthetic. Subcutaneous Temgesic was given postoperatively for pain management. Antibiotic (Duplocillin) was given intramuscularly 1 day before surgery and again 2 days postoperatively.

\section{Assessment of Edema}

The hind legs were shaved closely and leg circumference measurements were taken at a point $10 \mathrm{~cm}$ distal from the hock (tarsus). This landmark was highlighted preoperatively with a skin marker. Using a blank piece of umbilical tape, circumference measurements were taken daily in the first week after surgery and once a week after that until the animals were sacrificed. Between the hoof and hock/carpal joints, the sheep limb is fairly uniform in shape and diameter, eliminating the need for a circumference measurement at more than one point. Similarly, the forelimbs of 21 animals were shaved and measured throughout the experiment as controls at a point $10 \mathrm{~cm}$ distal to the carpal joint.

\section{Fluoroscopy}

Imaging was performed on 4 sheep at 8 weeks ( 1 animal, 2 limbs), 12 weeks ( 1 animal, 2 limbs) and 16 weeks after surgery (1 animal, 2 limbs), in addition to 1 control animal (2 limbs). Evans blue dye (1\% in saline) was injected subcutaneously to highlight the lymphatics prior to cannulation of an upstream popliteal prenodal vessel with a 26-gauge angiocatheter. An X-ray contrast medium (1-3 ml; Lipiodol, EZ-EM Canada, Therepex) was injected through the cannula, and a mobile fluoroscopy system (BV Pulsera, Philips) was used to visualize the lymphatic vessels around the popliteal region.

\section{Immunohistochemistry}

In 10 animals, the growth of new lymphatic vessels (lymphangiogenesis) was assessed at the nodal excision site. Briefly, 2 methods were used to identify the lymphatic vessels. First, 5(and 6)-carboxyfluorescein diacetate succinimidyl ester (CFDA- 


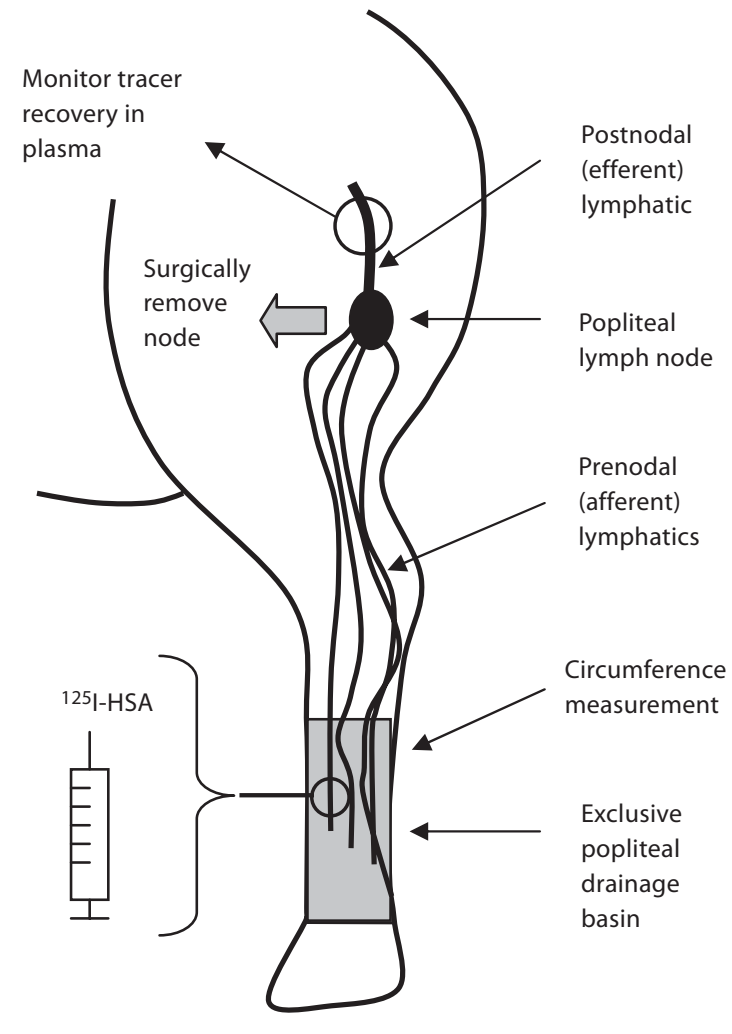

Fig. 1. Schematic illustrating essential features of the experimental model.

SE; Molecular Probes No. C-1157) was injected into an upstream prenodal lymphatic. This molecule diffuses passively into cells and remains nonfluorescent until its acetate groups are cleaved by intracellular esterases (it fluoresces green). This provided unequivocal identification of the lymphatics since the dye was introduced directly into the lumens of the vessels. In addition, the sections were co-stained with antibodies to the lymphatic endothelial receptor for hyaluronan (LYVE-1; tagged with Cy3 red).

Tissue blocks from the popliteal region were removed surgically. The samples were embedded in Tissue-Tek OCT compound, placed in a base mold and frozen immediately at $-80^{\circ} \mathrm{C}$. Sections (7 $\mu \mathrm{m}$ thick) were cut using a Leica cryostat (model Leica CM 3050S-3-1-1) and were placed on glass microscope slides. The slides were washed 5 times in phosphate-buffered saline (PBS; 0.1 $\mathrm{M}$ ) and blocked for $1 \mathrm{~h}$ at room temperature with $10 \%$ goat serum in PBS. After washing with PBS, the sections were incubated overnight at $4^{\circ} \mathrm{C}$ with 1:50 or 1:100 dilutions of rabbit, antihuman LYVE-1 primary antibody (Research Diagnostics Inc.). The next day, the sections were washed with PBS and incubated with 1:100 dilutions of goat, antirabbit IgG antibody tagged with Cy3 (Jackson Immuno Research). In controls, the primary antibody to LYVE-1 was omitted. Finally, the slides were mounted in aquapolymount and coverslipped.
Immunofluorescence microscopy was performed with a Zeiss Axiovert 100M laser scanning confocal microscope. The argon and helium/neon lasers were set to wavelengths of 488 and 543 $\mathrm{nm}$ for excitation of CFDA-SE and Cy3, respectively. When both techniques were combined in the same tissue sample, the lymphatic vessels appeared yellow-orange.

Quantitative Assessment of Protein Transport to Plasma

The main role of the lymphatic vessel is to absorb extravasated vascular derived protein from the interstitial spaces and return it to the venous circulation. Therefore, the ability to transport a known mass of radiolabeled albumin to plasma provides a quantitative measure of the lymph transport effectiveness of a given lymphatic network. A schematic illustrating the features of the experimental design is provided in figure 1 .

Quantitative studies were performed at 8, 12 and 16 weeks following popliteal nodectomy as well as in a node-intact group.

The animals were fasted and anesthesia was induced as previously described. The hind limbs were shaved and a final circumference measurement was made at the landmark described above. A heparinized neckline was inserted into the jugular vein and secured for collecting blood samples throughout the experiment. Evans blue dye (1\% in saline) was injected under the skin to highlight the lymphatics. When injected subcutaneously, the dye binds to protein and readily enters absorbing lymphatics. It is distributed rapidly through the lymphatic network and outlines the collecting vessels clearly. An incision was made through the skin and subcutaneous tissues over the lower lateral aspect of the hind limb, extending distally from the hoof to expose several prenodal lymphatic vessels in close proximity to the lateral saphenous vein. With the aid of magnifying loups, a single vessel was dissected free from the surrounding tissues and cannulated with a 26-gauge angiocatheter. This was secured with 4.0 silk ties and a clamp was applied distally to prevent backflow.

Saline $(100 \mu \mathrm{l})$ was injected slowly over $30 \mathrm{~s}$ to check for any leaks at the cannulation site and observe the flushing of the Evans blue dye in the lymphatic vessel. This process ensured the correct placement of the cannula. Radiolabeled human serum albumin ( ${ }^{125} \mathrm{I}-\mathrm{HSA} ; 2 \mathrm{mg}$ in a $200-\mu \mathrm{l}$ volume) was injected into a prenodal lymphatic vessel over a 60 -second period with a 250 - $\mu$ l Hamilton syringe and flushed with $100 \mu \mathrm{l}$ of saline over $30 \mathrm{~s}$. The cannula was then capped. Swabs were taken from around the tip of the cannula as well as the injection site and later analyzed in a gamma counter to indicate whether or not there was a leak or spill of the radioactive tracer.

Blood samples were taken from the neckline to monitor the recovery of radioactivity in the blood over a period of $4 \mathrm{~h}$. Samples were taken at 0,15 and $30 \mathrm{~min}$ and then every $30 \mathrm{~min}$ up to $4 \mathrm{~h}$. The animals were sacrificed at the end of the experiment with 20 $\mathrm{ml}$ of Euthanyl administered intravenously.

The concentrations of the radioactive protein tracer in plasma (counts per minute per milliliter) were divided by the amount injected to arrive at the percent injected per milliliter, which was plotted over time. However, once a protein tracer enters the blood, it refilters back into the various tissues of the body and hence tracer recoveries are inherently underestimated. An albumin elimination rate $\left(\mathrm{K}_{\text {exp }}\right)$ has been defined in previous experiments [8] and was used in a mass balance equation (equation 1) to reflect more accurately the ability of the lymphatic system to return protein to blood. The mass balance equation outlined 
below was used to estimate a single averaged mass transport rate:

$$
B_{i n}=\frac{\left[C_{P}\left(t_{f}\right) \exp \left(K_{\exp } t_{f}\right)-C_{P}(0)\right]\left(K_{\exp } V_{P}\right)}{\exp \left(K_{\exp } t_{f}\right)-1}
$$

$B_{\text {in }}$ (blood in) $=$ the mass transport rate (counts per minute per hour), which was averaged from time 0 to time $t_{f}$ (duration of experiment), which in our case was $4 \mathrm{~h}$. The values for $\mathrm{B}_{\text {in }}$ derived from equation 1 were divided by the total radioactivity injected to give the percent injected per hour. $\mathrm{C}_{\mathrm{P}}\left(\mathrm{t}_{\mathrm{f}}\right)=$ the concentration of the tracer at $4 \mathrm{~h} ; \mathrm{C}_{\mathrm{P}}(0)=$ the concentration of the tracer at time 0 ; $\mathrm{K}_{\text {exp }}=$ is the coefficient of elimination of tracer from plasma. Since our previous experience indicated that this coefficient did not differ significantly between animals of various ages and weights, an average value was derived from 41 animals used in previous studies [8-11]. Since the volume of distribution of the tracer (plasma volume, $\mathrm{V}_{\mathrm{P}}$ ) would differ between animals, we adjusted the plasma recoveries to reflect this. Based on data derived in previous studies by our group [8-11], we plotted the plasma volumes derived from 41 animals against their weights. We used a regression analysis of these data (equation 2) to calculate a plasma volume in each sheep based on the following equation:

$$
y=21.77 x+649.68
$$

\section{Statistical Analysis}

All data are expressed as means \pm SEM. The data were analyzed with a one-way ANOVA followed by contrasts back to baseline using a one-sided Dunnett's t test. We considered $\mathrm{p}<0.05$ as significant.

\section{Results}

\section{Development of Lymphedema}

The removal of a single popliteal lymph node resulted in edema formation in the lower hind limbs of all animals. An example is illustrated in figure $2 a$. Figure $2 b$ displays the averaged data. On day one after surgery, the average increase in leg circumference was $19.5 \%$, on day two $29.1 \%$ and on day three $33.8 \%$ (the peak of the response). The edema declined over time but in most animals did not subside completely and remained elevated until the sheep were sacrificed (up to 16 weeks after surgery). In 3 sheep, the edema subsided completely before the end point of the study. All of the control forelimbs which were measured showed no increase in circumference. All animals were ambulatory during the course of the experiments.

\section{Assessment of Lymphatic Function}

When ${ }^{125}$ I-HSA was injected into an upstream prenodal popliteal lymphatic vessel in control limbs, it entered plasma with peak concentrations being achieved
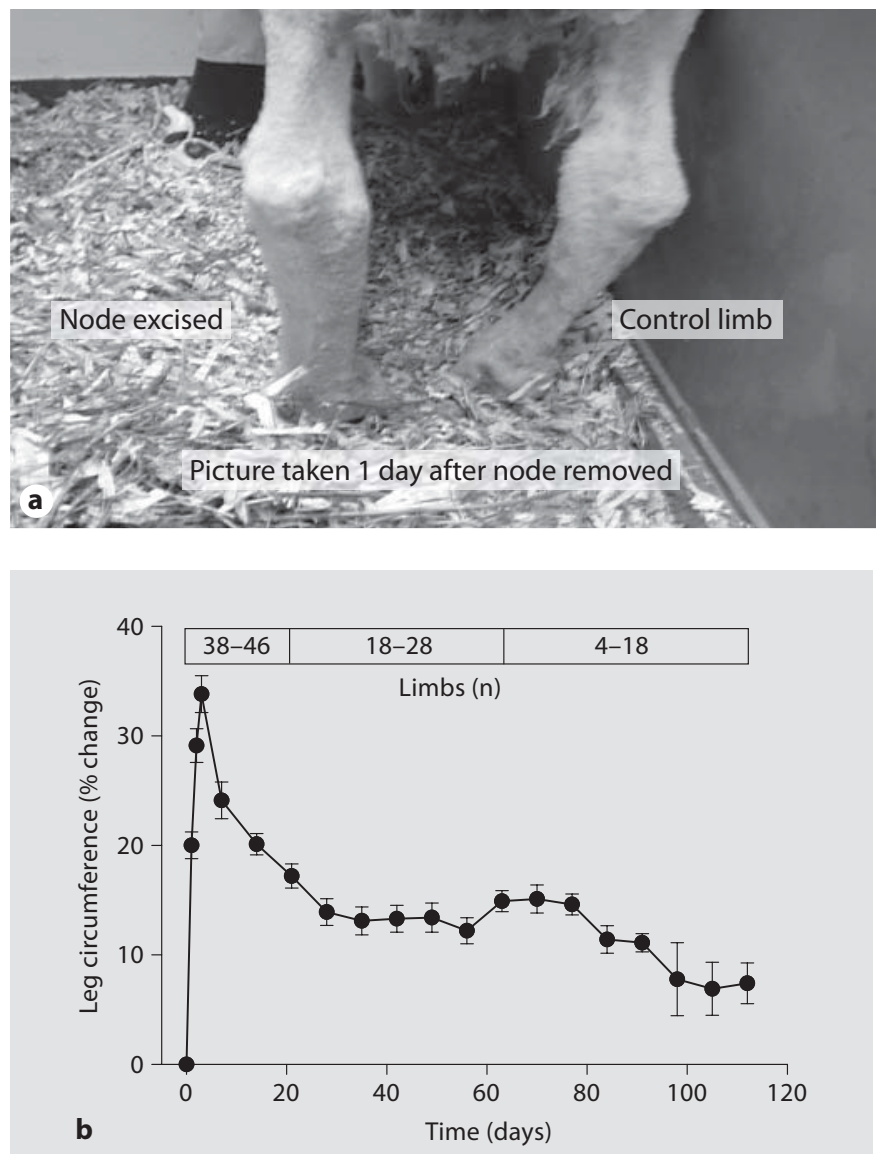

Fig. 2. Lymphedema produced after the removal of the popliteal lymph node. a Example of edema generation following lymph node excision in comparison with a control limb. b Quantification of edema after lymph node excision expressed as percentage change from presurgical levels. The numerals at the top of the graph illustrate the number of limbs that were assessed at various times following lymph node excision.

approximately $1 \mathrm{~h}$ after injection (fig. 3). Measurement of plasma levels at 8,12 and 16 weeks following lymph node excision revealed lower blood concentrations.

Figure 4 illustrates the averaged tracer mass transport rates over $4 \mathrm{~h}$ calculated from equation $1\left(\mathrm{~B}_{\text {in }}\right)$. A one-way ANOVA revealed that the groups were significantly different. The values obtained at $8(10.6 \pm 1.5), 12(14.4 \pm$ $1.0)$ and 16 weeks after surgery $(13.9 \pm 1.0)$ were less than that noted for the intact limbs $(17.2 \pm 0.6)$ but only the data at 8 weeks reached statistical significance with Dunnett's t test.

These data suggest that pre- and postnodal lymphatic vessels had regenerated to a considerable extent, since the 
Fig. 3. Quantification of lymphatic function. Radioactive albumin was injected into a prenodal lymphatic vessel and the concentration of the tracer was monitored in plasma for $4 \mathrm{~h}$ after injection in 4 groups of animals, i.e. the control group $(n=7)$ and $8(n=7), 12(n=7)$ and 16 weeks $(n=$ 6) after surgery.

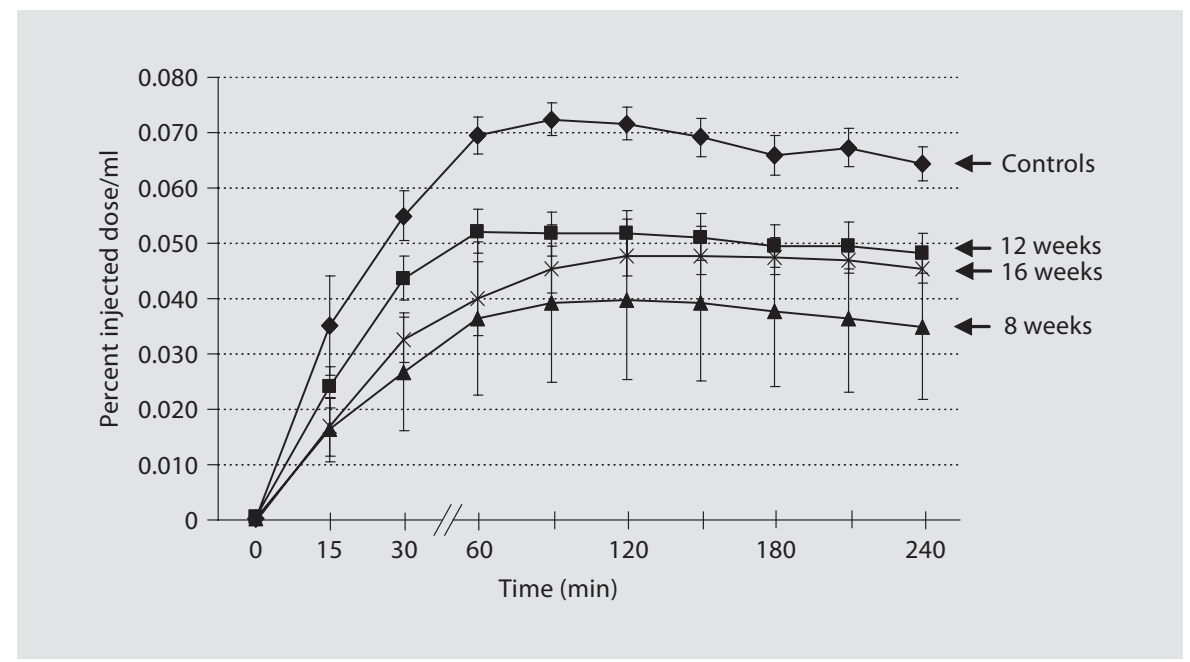

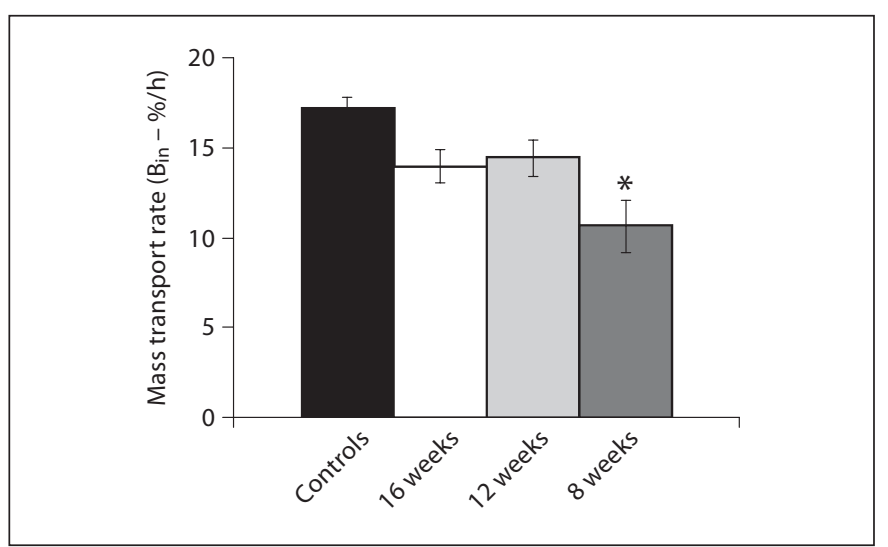

Fig. 4. Quantification of lymphatic function. Equations 1 and 2 were used to calculate an average mass transport rate for each group of animals, i.e. controls $(n=7)$ and $8(n=7), 12(n=7)$ and 16 weeks $(n=6)$ after surgery. A one-way ANOVA revealed that the groups were significantly different. The values obtained at 8 , 12 and 16 weeks after surgery were less than those noted for the intact limbs, but only the data at 8 weeks reached statistical significance with Dunnett's t test $\left(^{*}\right)$.

entry of tracer into plasma showed that some lymph continuity had been reestablished. Indeed, at 12 and 16 weeks after surgery, lymphatic function had returned to approximately $80 \%$ of the level observed in control limbs. At 8 weeks after surgery, only about $60 \%$ of lymph transport had been restored.

\section{Fluoroscopy and Immunohistochemistry}

Fluoroscopic analysis at 8,12 and 16 weeks after nodal excision confirmed that some degree of fluid continuity had been established between the prenodal and postnodal lymphatic vessels in the absence of the lymph node. The contrast agent injected into one of the prenodal lymphatics in the lower limb could be observed in the relatively large duct that would normally collect lymph from the popliteal node (postnodal vessel; example illustrated in fig. 5a). Small lymphatic vessels were observed in the nodal excision area. The possibility that these vessels were newly formed lymphatics attempting to bridge the gap left by the absent node was supported by immunohistochemistry.

We observed colocalization of LYVE-1 (red) and CFDA-SE (green), indicating that lymphatic vessels were present at the area vacated by the popliteal lymph node. An example is provided in figure 5b. The newly formed lymphatics observed at 12 weeks after surgery were variable in size, but at the upper end were about 40 $\mu \mathrm{m}$ in diameter. These vessels were the conduits that connected the originally placed pre- and postnodal lymphatics and contributed to the restoration of lymph transport that was observed in the tracer and fluoroscopic studies.

\section{Discussion}

In this paper, we report the development of a sheep lymphedema model which permits the integration of clinical edema with quantifiable lymphatic transport function. The data suggest that the removal of a single lymph node produces a lymph drainage deficit in the affected limb. We obtained evidence of lymphatic vessel regeneration in the area originally occupied by the lymph 

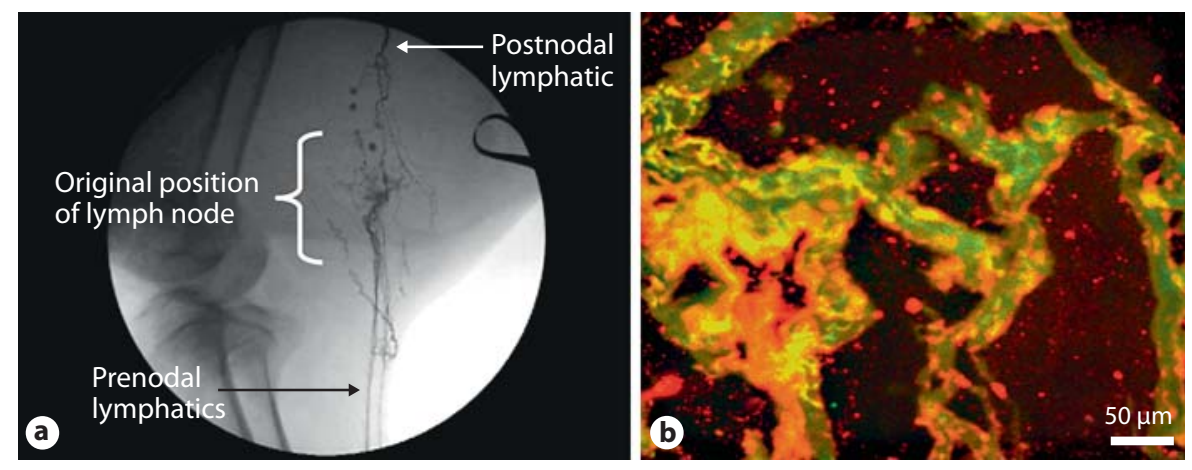

Fig. 5. Morphological evidence that some regeneration of lymphatic vessels has occurred following popliteal lymph node excision. a Fluoroscopy performed at 12 weeks after lymph node removal illustrating some degree of continuity between the lymphatic vessels that were originally pre- and postnodal to the popliteal lymph node. $\mathbf{b}$ Confocal microscopy image of regenerated postnodal lymphatics in the nodal excision site. Tissue sections were prepared as described in Materials and Methods. For unequivocal identification of these vessels, a nonspecific cell dye
CFDA-SE (green) was infused into an upstream prenodal lymphatic and, at the same time, the tissues were stained with antibodies to the lymphatic endothelial marker LYVE-1 (red). The stained sections appeared yellow-orange, indicating coexistence of the 2 stains. At 12 weeks, an irregular network of small interconnecting lymphatics could be observed in the area originally occupied by the popliteal lymph node. We presume that all vessels in this image are newly formed in response to the removal of the node. node, and by $12-16$ weeks after node excision about $80 \%$ of lymphatic function had been reestablished. At this time, clinical edema was still evident in most animals.

\section{Animal Models of Lymphedema}

The development of animal models to simulate human lymphedema has been challenging due to the remarkable regenerative capacity of the lymphatics and the formation of collateral pathways around the site of obstruction. In some cases, extensive surgical manipulation is required to elicit edema [reviewed in 6]. As an example, in the studies of Olszewski [12], lymphedema was achieved in dogs by excising a circumferential strip of skin, subcutaneous tissue and fascia from the upper region of the thigh and the periosteum of the femur was stripped off. This was followed by a second procedure involving the removal of the popliteal lymph node. With varying degrees of surgical manipulation, lymphedema models have been applied to several anatomical locations and species. Commonly, a significant tissue mass is removed with attendant dissection of lymphatic vessels and nodes. Popular models include the hind limb models in dogs [12] and rats $[13,14]$, rabbit ear models $[3,15]$ and rodent tail models [16-18]. In some animal models, radiation is applied in addition to surgical tissue resection $[19,20]$.

One of the limitations of many of the aforementioned models is difficulty in quantifying lymphatic function. Histological assessment of molecular markers is com- monly used to assess the presence or absence of lymphatic vessels, with attempts to correlate these findings with the clinical status of the animal [21]. This approach has been popular in murine models, but the functionality of the newly formed vessels is difficult to determine with immunohistochemistry. The tissue injection of various contrast agents has been used to identify newly developing lymphatic networks. For example, the intradermal injection of FITC-dextran combined with fluorescence microlymphangiography has been used to assess the formation of new lymphatic vessels and provides some opportunity to quantify lymph transport parameters [17, 18]. In general terms, however, the functional impact of lymphangiogenesis on fluid transport remains a significant challenge in most lymphedema models, especially those that employ small species. In this regard, studies in sheep have several advantages.

\section{Advantages of a Sheep Lymphedema Model}

The anatomical dimensions of the sheep lymphatic system provide an opportunity to quantify lymphatic function and offer a realistic framework for the development of therapeutic measures and methods of administration that are 'human-sized' in perspective.

Especially important is the ability to monitor the transport of a known mass of radiolabeled albumin to plasma as this provides a useful measure of the lymph transport effectiveness of a given lymphatic network. Un- 
der normal conditions, molecules with molecular weights $>6,000 \mathrm{Da}$ injected into a limb lymphatic are almost completely recovered in thoracic duct lymph [22]. Therefore, following the injection of ${ }^{125} \mathrm{I}$-HSA into prenodal lymphatic vessels, one would expect that the mass transport rate of this protein tracer to plasma would reflect the integrity of the lymphatic network in question. Indeed, we were able to observe measurable differences in the plasma recoveries after lymph node excision compared to control limbs.

A significant benefit to studies of the popliteal lymphatic system is that the pre- and postnodal popliteal ducts are easy to identify and some of these are of sufficient size for cannulation. In sheep, usually only 1 vessel drains the node (in rare occasions there are 2). Multiple prenodal ducts (6-12) enter the popliteal lymph node at various locations along the convex portion of the node [23]. Since there is no known collateral lymphatic circulation in this area, we expected that removal of the popliteal lymph node would affect all lymph flow from the lower hind limb and induce edema, a prediction borne out by the experiments in this report. The ability to disrupt the regional tissue drainage in a limb by removing a lymph node makes this model especially relevant to postsurgical lymphedema such as that which occurs in breast cancer patients.

In addition to the aforementioned issues, sheep lymphatics are known to regenerate after injury and in response to surgical node removal [24-26]. Recent data indicate that sheep lymphatic endothelial cells express the requisite molecular markers for identification and that vessel regeneration is characterized by the upregulation of factors known to be associated with lymphangiogenesis in other animal species [26].

In cancer patients, the removal of one or more lymph nodes often gives rise to acute edema, which resolves successfully. It is, of course, the chronic form of edema that occurs in a subgroup of patients that is most problematic. In this regard, there is no agreed upon time beyond which one considers the edema to be chronic. For practical reasons, we did not follow the animals beyond a 16-week period. It is possible, therefore, that the average edema in our animals would have resolved if the sheep had been permitted to survive for longer periods. Nonetheless, the model is suitable for the measurement of lymphatic-related physiological changes over a reasonable period of time.

\section{Questions Related to Lymphedema Development}

There are many questions in the lymphedema field that have to be addressed before new, rational therapies for this condition can be developed. As one example, an assumption has always been made that the major impediment to lymph transport in postsurgical lymphedema patients is due entirely to lymphatic vessel injury and the inability of the regenerating vessels to remove tissue fluid adequately. However, lymphatic vessels are damaged routinely in surgical procedures and the acute, postsurgical edema usually resolves rapidly as the lymphatics regenerate. Certainly, we observed new lymphatic vessel growth at the nodal excision site, which would agree with other accounts in the literature. In rabbits, the removal of a popliteal lymph node results in lymphangiogenesis and the formation of new ducts and collateral vessels as early as 4 weeks after node removal [27]. Given the robust lymphatic response to injury, one might speculate that generation of lymphedema may also involve other anatomical elements and mechanisms. In this regard, it would seem that there might be something special about the removal of a lymph node. Indeed, it is clear that the removal of lymph nodes greatly increases the chance of developing lymphedema, especially if this is combined with radiotherapy [7].

One factor that might be worth considering is that the lymph node appears to anatomically separate the lymphatic system into higher (prenodal) and lower (postnodal) pressure areas [22]. This is due to the fact that proteinfree fluid is absorbed from the lymph into the blood capillaries in the nodes to establish an equilibrium of Starling forces across the lymph-blood barrier [28-30]. An important component of the lymph transport system is the ability of pre- and postnodal lymphatics to contract and pump lymph. From the studies of Ikomi et al. [27], it would seem that the regenerating lymphatic vessels reacquire a contractile phenotype 4 weeks after nodal excision. However, the removal of the lymph node may lead to higher intralymphatic pressures in the downstream vessels, and this would force these vessels to function under nonoptimal conditions.

Another issue may relate to lymph flow resistance. The resistance of large lymphatic vessels is generally believed to be low and of little physiological significance [22]. In contrast, lymph nodes are known to provide resistance to lymph transport and this has been estimated to be 50 200 times greater than that provided by the lymph trunks [31]. One might expect, therefore, that the removal of a node would reduce flow resistance. However, there is some evidence that the resistance to flow increases after the removal of a lymph node, at least over a relatively short period [25]. The new lymphatics that form in response to nodal excision exist as an irregular network of 
small vessels and it is possible that this arrangement provides an impediment to flow. It is, of course, possible that resistance will change over time as the new lymphatic network matures and remodels in an attempt to produce a configuration that may be more amenable to effective lymph transport.

In any event, under normal circumstances, limb compliance and lymphatic function work to maintain tissue water levels at some optimal level. Guyton et al. [32] have described the concept of a 'safety factor' that works to prevent increases in interstitial fluid volume. The normal negative limb interstitial fluid pressures provide some hydrostatic buffering effect (reserve capacity), as interstitial fluid pressure must rise before edema develops. It is more than likely that each insult to the lymphatic-lymph node axis serves to consume some of this reserve and move the limb closer to the point at which clinical edema may occur. In the data presented here, regeneration of the lymphatic vessels over a 12- to 16 -week period obviously helped to restore a sizeable portion (about $80 \%$ ) of the lost lymph transport capacity and no doubt facilitated the resolution of a significant portion of the edema. However, in terms of risk to the patient, one might speculate that lymphangiogenesis by itself may not be able to restore the full reserve capacity of the system. There is some evidence that the newly regenerating lymphatic network provides an increased resistance to lymph flow [25]. Additionally, the ability of the limb to remove tissue fluid may still be compromised (even at a subclinical level) due to the removal of the nodes and the loss of their ability to 'depressurize' the lymphatic system. Added to this, one would expect that radiotherapy could consume additional functional reserve. Taken together, these changes would make the lymphatic system less effective at transporting interstitial fluid and could lead to edema formation in the cancer patient.

In summary, in this report we describe an experimental model in sheep that permits the quantitation of edema and lymphatic function over time. The basis of this approach is the removal of a single lymph node. Our longterm objective is to investigate the physiological parameters that lead to lymphedema formation and develop new strategies to treat or prevent postsurgical edema.

\section{Acknowledgements}

This project is funded by the Canadian Breast Cancer Foundation-Ontario Chapter. We also thank the Advanced Regenerative Tissue Engineering Centre, Sunnybrook Health Sciences Centre, for support of this project. We are very grateful to Ms. Dianna Armstrong, Ms. Sara Moore and Ms. Azadeh Jila for excellent technical assistance during these studies. Additionally, we wish to thank M. Katic (Department of Research Design and Biostatistics, Sunnybrook Health Sciences Centre) for help in the computational analyses of the data. D.T. was the recipient of a Fellowship award from The Henrietta Banting Foundation (Plastic Surgery Division, Department of Surgery).

\section{References}

1 Armer JM, Heppner PP, Mallinckrodt B: The secret epidemic: lymphedema. J Women Cancer 2001;3:35-41

2 Badger C, Preston N, Seers K, Mortimer P: Physical therapies for reducing and controlling lymphoedema of the limbs. Cochrane Database Syst Rev 2004;4:CD003141.

- 3 Szuba A, Skobe M, Karkkainen MJ, Shin WS, Beynet DP, Rockson NB, Dakhil N, Spilman S, Goris ML, Strauss HW, Quertermous T, Alitalo K, Rockson SG: Therapeutic lymphangiogenesis with human recombinant VEGF-C. FASEB J 2002;16:1985-1987.

-4 Saaristo A, Veikkola T, Tammela T, Enholm B, Karkkainen MJ, Pajusola K, Bueler H, YlaHerttuala S, Alitalo K: Lymphangiogenic gene therapy with minimal blood vascular side effects. J Exp Med 2002;196:719-730.

5 Yoon YS, Murayama T, Gravereaux E, Tkebuchava T, Silver M, Curry C, Wecker A, Kirchmair R, Hu CS, Kearney M, Ashare A, Jackson DG, Kubo H, Isner JM, Losordo DW: VEGF-C gene therapy augments postnatal lymphangiogenesis and ameliorates secondary lymphedema. J Clin Invest 2003;111:717725.

6 Piller NB, Clodius L: Experimental lymphoedema: its applicability and contribution to our clinal understanding; in Johnston M (ed): Experimental Biology of the Lymphatic Circulation. Amsterdam, Elsevier Science, 1985, pp 189-230.

-7 Ryttov N, Holm NV, Qvist N, Blichert-Toft M: Influence of adjuvant irradiation on the development of late arm lymphedema and impaired shoulder mobility after mastectomy for carcinoma of the breast. Acta Oncol 1988;27:667-670.

8 Boulton M, Flessner M, Armstrong D, Hay J, Johnston M: Lymphatic drainage of the CNS: effects of lymphatic diversion/ligation on CSF protein transport to plasma. Am J Physiol 1997;272:R1613-R1619.

-9 Boulton M, Armstrong D, Flessner M, Hay J, Szalai JP, Johnston M: Raised intracranial pressure increases CSF drainage through arachnoid villi and extracranial lymphatics. Am J Physiol 1998;275:R889-R896.

10 Boulton M, Flessner M, Armstrong D, Hay J, Johnston M: Determination of volumetric cerebrospinal fluid absorption into extracranial lymphatics in sheep. Am J Physiol 1998;274:R88-R96.

- 11 Boulanger B, Yuan Z, Flessner M, Hay J, Johnston M: Pericardial fluid absorption into lymphatic vessels in sheep. Microvasc Res 1999;57:174-186.

12 Olszewski W: Induction of experimental lymphatic edema (in Polish). Pol Przegl Chir 1967:39:926-929.

13 Piller NB, Casley-Smith JR: The effect of coumarin on protein and PVP clearance from rat legs with various high protein oedemas. Br J Exp Pathol 1975;56:439-443.

14 Liu Y, Fang Y, Dong P, Gao J, Liu R, Tian H, Ding Z, Bi Y, Liu Z: Effect of vascular endothelial growth factor C (VEGF-C) gene transfer in rat model of secondary lymphedema. Vascul Pharmacol 2008;49:44-50. 
15 Huang GK, Hsin YP: An experimental model for lymphedema in rabbit ear. Microsurgery 1983;4:236-242.

- 16 Slavin SA, Van den Abbeele AD, Losken A, Swartz MA, Jain RK: Return of lymphatic function after flap transfer for acute lymphedema. Ann Surg 1999;229:421-427.

17 Swartz MA, Boardman KC Jr: The role of interstitial stress in lymphatic function and lymphangiogenesis. Ann NY Acad Sci 2002; 979:197-210.

- 18 Boardman KC, Swartz MA: Interstitial flow as a guide for lymphangiogenesis. Circ Res 2003;92:801-808

19 Das SK, Franklin JD, O’Brien BM, Morrison WA: A practical model of secondary lymphedema in dogs. Plast Reconstr Surg 1981;68: 422-428.

-20 Chen HC, O’Brien BM, Rogers IW, Pribaz JJ, Eaton CJ: Lymph node transfer for the treatment of obstructive lymphoedema in the canine model. Br J Plast Surg 1990;43:578586.
21 Cunnick GH, Jiang WG, Gomez KF, Mansel RE: Lymphangiogenesis quantification using quantitative PCR and breast cancer as a model. Biochem Biophys Res Commun 2001; 288:1043-1046.

22 Aukland K, Reed RK: Interstitial-lymphatic mechanisms in the control of extracellular fluid volume. Physiol Rev 1993;73:1-78.

23 Heath T, Brandon R: Lymphatic and blood vessels of the popliteal node in sheep. Anat Rec 1983;207:461-472.

24 Andrade WN, Johnston MG, Hay JB: The relationship of blood lymphocytes to the recirculating lymphocyte pool. Blood 1998;91: 1653-1661.

25 Kim C, Li B, Papaiconomou C, Zakharov A, Johnston M: Functional impact of lymphangiogenesis on fluid transport after lymph node excision. Lymphology 2003;36:111-119.

26 Jila A, Kim H, Nguyen VP, Dumont DJ, Semple J, Armstrong D, Seto E, Johnston M: Lymphangiogenesis following obstruction of large postnodal lymphatics in sheep. Microvasc Res 2007;73:214-223.
27 Ikomi F, Yokoyama Y, Ogiwara N, Sasaki K, Mizuno R, Ohhashi T: Recanalization of the collecting lymphatics in rabbit hind leg. Microcirculation 2006;13:365-376.

$\checkmark 28$ Adair TH, Moffatt DS, Paulsen AW, Guyton AC: Quantitation of changes in lymph protein concentration during lymph node transit. Am J Physiol 1982;243:H351-H359.

29 Adair TH, Guyton AC: Modification of lymph by lymph nodes. II. Effect of increased lymph node venous blood pressure. Am J Physiol 1983;245:H616-H622.

30 Adair TH, Guyton AC: Modification of lymph by lymph nodes. III. Effect of increased lymph hydrostatic pressure. Am J Physiol 1985;249:H777-H782.

>31 Papp M, Makara GB, Hajtman B: The resistance of in situ perfused lymph trunks and lymph nodes to flow. Experientia 1971;27: 391-392.

32 Guyton AC, Granger HJ, Taylor AE: Interstitial fluid pressure. Physiol Rev 1971;51:527563. 